\title{
Psychiatry 2050: from younger psychiatrists' perspective
}

\author{
This article was published in the following Dove Press journal: \\ Neuropsychiatric Disease and Treatment \\ 7 May 2014 \\ Number of times this article has been viewed
}

\section{Tariq Mahmood Hassan \\ Wasif Habib \\ Mir Nadeem Mazhar \\ Tariq Munshi}

Department of Psychiatry, Queen's University, Kingston, ON, Canada
Correspondence: Tariq Mahmood Hassan Providence Care Mental Health Services, 752 King Street West, Kingston, Ontario, K7L 4X3, Canada

Email hassant@queensu.ca
There have been various opinion pieces on predicting the future of psychiatry and addressing its different domains. This editorial addresses the topic from the vantage point of neuroscientific inquiry. The Diagnostic and Statistical Manual of Mental Disorders, Fifth Edition (DSM 5) however continues with the tradition of its predecessor (DSM 4 text revision [TR]), addressing most diagnoses with descriptive phenomenology as opposed to attempting to change diagnoses based on causative phenomenology or response to treatment. Advances in genomics and imaging, with time, will hopefully help shape psychiatric diagnoses and classifications with a primary basis on morphology. This may in turn help improve the recruitment of academic psychiatrists to the field. In doing so, the profession will gain respect amongst its peers in other disciplines of medicine and cement its future.

Over the years many opinion pieces addressing the future of psychiatry have been published in a variety of journals. The literature so far has delved into strategies to increase the interest in the field by strengthening the relationships between psychiatry and other medical disciplines, as well as the future of the profession itself. This editorial does not stray too far from that concept but does attempt to address this topic by dealing with a parallel niche of neuroscientific inquiry.

Mental disorders are now among the largest sources of medical disability worldwide. According to the World Health Organization, neuropsychiatric disorders account for at least $20 \%$ of the global burden of illness-related disability, and all represent complex disorders of brain function. ${ }^{1}$ The context is relevant as the basis of clinical neuroscience emanating from studies of etiology and pathophysiology of brain disorders directly impacts and influences the advances in assessment, treatment and prevention of brain disorders. We believe that with time psychiatric disorders will be conceptualized as disorders of the brain and advances in assessment, treatment, and prevention are likely to originate from studies of etiology and pathophysiology based in clinical neuroscience.

There are several critical future challenges to psychiatry as a profession. One of these challenges is a diminishing confidence in the knowledge base regarding diagnosis and management, and the perceived lack of a coherent theoretical basis. This has significant negative implications for research funding opportunities and attracting research minded clinicians compared to the big hitters of medicine, surgery, and cancer research. Although mental health funding in most developed western countries has increased it is still much smaller in comparison to the other medical and surgical 
specialties, with the public health burden of mental disorders growing exponentially.

The latest version of the DSM 5 came into effect in May 2013 following a 14 year revision process. There seemed to have been a nervous anticipation that morphological basis to diagnosis may make a bold entrance into the DSM 5 albeit a gradual one. DSM 5 however continued with the tradition of DSM 4 TR further focusing on addressing the diagnostic criteria for making a diagnosis, rather than attempting to alter diagnoses based on etiological processes. ${ }^{2}$ The diagnosis of 'schizophrenia' remains the same for now, 100 years on. There is emerging evidence to tailor treatment options based on genetic profiles. In a study on bipolar patients of Han Chinese descent, it was found that those who had genetic variations in the glutamate decarboxylase like protein 1 were associated with greater response to lithium maintenance for bipolar I disorder. ${ }^{3}$ Farooq et $\mathrm{al}^{4}$ have proposed a new paradigm in classifying subtypes of schizophrenia based on treatment responses. There are also concerns that although the DSM was created to provide a comprehensive system for diagnosing and evaluating psychiatric patients, it has been given almost total authority in training programs and health care delivery systems. A criticism is that students are taught to memorize the DSM rather than learn the complexities of psychopathology, with history taking being reduced to a DSM checklist. ${ }^{5}$

A part of this editorial should focus on altering the current system of classification by incorporating morphological features as diagnostic criteria, which are in development. ${ }^{6}$ Psychiatric diagnostic practice has evolved under the biomedical model till now. We would suggest however that psychiatric classification systems should facilitate communication of information to promote understanding by colleagues and patients, by including descriptions of pathogenesis, prognosis, and treatment choices, as well as morphological features.

After a period of uncertainty and confusion psychiatric genetics research has started to yield results. The prospect that genomic research will change psychiatry in the future remains ongoing. ${ }^{7}$ Through big international collaborative efforts there are more than 60 established genetic variants which have strong association with schizophrenia, for example (results presented in the World Congress of Psychiatric Genetics, Hamburg 2012). ${ }^{8}$ The Human Genome Project completed in 2003, ${ }^{9}$ promises greater insight into understanding and functions of the human genome. This information is freely available on the Internet and has allowed a vast number of new genes to be explored and studied, teaching us how the brain develops and functions. The areas of neuroscience and genomic research are two areas of science fundamental to psychiatry that have undergone significant advances in the past two decades. ${ }^{10}$ However, their impact on practice has been limited, while during the same period of time, the public health burden of mental disorders has grown exponentially due to the complexity of these disorders but unfortunately there are no short cuts. Genomics has only now advanced to stages which will almost certainly change the way psychiatric patients are treated in future and may include more targeted therapies for schizophrenia and autism. We may also see genetic tests that could predict pharmacological treatment response or vulnerability to a particular adverse effect. However, the biggest dividend will be a better understanding of the underlying biological mechanisms which lead to symptoms.

The current system of classification by incorporating morphological features as diagnostic criteria is in development. ${ }^{2}$ Historically the phenomenological patterns of illness presentation were the key to most, if not all psychiatric diagnoses. We are moving away from classifying diagnoses as either "organic" or "functional", thereby highlighting a greater understanding of the morphological characteristics, albeit predominantly at a molecular level, of major mental illnesses. In future this will facilitate communication of information to promote a clearer understanding by our medical and surgical colleagues as well as patients, by linking descriptions of pathogenesis, prognosis, and treatment choices with morphological features.

For the purpose of this editorial piece, it is our opinion that the perception of psychiatry in the eyes of the public and other medical disciplines will be in part contributed to by the further understanding of underlying neuropathological processes. On one hand the anti-stigma public campaigns supporting the mentally ill have increased in recent years, while on the other, governments fail to prioritize mental illness when compared to the other medical disciplines. Psychiatry must therefore be communicated in a language that is neither vague nor ambiguous. The emergence of functional neuroimaging that permits the visualization of distinctive neurological activity that helps in distinguishing between abnormal and normal neurological functioning in the context of psychiatric illness in part facilitates the discussion of psychiatry being at par (or close to) other disciplines and not solely abstract in its language.

If we are proponents of psychiatric illness as neuropathological in etiology, as we are, we must adopt the teaching of neurology, neuropathology, and genetics as a standardized part of the psychiatric curriculum taught in medical schools 
as well as psychiatric training programs. This would ideally be done without compromising the psychiatric profession's expertise in interpretation of the interplay between behavior and emotion.

The aim is keep psychiatry's uniqueness in focus with all its complexities but at the same time to provide further clarity on its morphological processes.

At present there are significant limitations in our understanding of the genetic processes underlying many, if not most, psychiatric disorders. There has been tremendous progress made in localizing the specific genes that may play a role in the development of these disorders, but it is not yet clear how this will translate into clinically useful diagnostic tools or markers. While the time has not yet arrived for the clinical utility of these discoveries, the potential is there for using this data in order to estimate risks, identify patterns of inheritance, and adopt targeted interventions thereof.

Genetic epidemiological research and twin studies have demonstrated that the environment plays an important role along with genetic predisposition in the pathology of psychiatric illness.

The new focus is now on epigenetics and gene expression and the effect of the individual's environment on these two factors. When we speak of environmental factors, we include life experiences and circumstances, psychosocial stressors, diet and nutrition, exposure to chemicals both harmful and non-harmful light exposure, rates of metabolism, and hormonal influences as they relate to different psychodevelopmental and neurodevelopmental stages. It is hoped that the study of environmental factors and the role in gene expression will be further understood through the up-and-coming field of psychiatric genomic research.

At present there is great interest in the use of biological markers as indicators of psychiatric illness. At present there are no biological markers available for clinical use for diagnosis of psychiatric illnesses. The aim is to hopefully discover biological markers that can help diagnose psychiatric illnesses reliably and consistently, allow interventions to be personalized, and appropriately categorize individuals with these illnesses. This will ultimately depend on the translation of new findings and research to clinical practice.

As mentioned previously, the conceptualization of psychiatric illness as a neuropathological diseases mandates that the current and the new generations of psychiatrists and trainees require more understanding of neuropathology within the psychiatric realm and better training in neurological disorders.
In this editorial, we have already mentioned the advances in genomics and psychiatry as a neuroscience. This endorses psychiatry to be well positioned to take advantage of these advances and to build on them. Psychiatry has in fact become a popular specialty for medical students and residents who have an interest in research. This training involves intensive research experience and hopefully leads on to independent research in either the laboratory or clinical setting. Many of these researchers are coming from a neuroscience background that is in keeping with the above discussion about transforming psychiatry into a clinical neuroscience.

Medical students and psychiatry trainees drawn to careers in academic psychiatry should consider the research resources available in the psychiatric residency programs at which they interview. Medical students who undertook research during medical school anticipating continuing research in addition to obtaining clinical training in psychiatry, will most likely pick a program that can provide both opportunities.

In conclusion, it is our opinion that the future of psychiatry lies in the fields of biology, neuroscience, genomics, and research. It is important to remember however that in many of these areas progress and research are still in their infancy. A further understanding of the interplay of biology, genomics, and neuroscience will help clarify some of the mysteries of psychiatric diagnoses and morbidity. Classification systems and definitions of disorders will primarily incorporate neuroscience as a guide. The promise for psychiatry is dually paved with optimism and mystery and affords our successors the opportunity to revolutionize the prevention, diagnosis, and treatment of mental disorders.

\section{Acknowledgment}

The authors would like to thank Dr Mohammed Ayub and Dr Khalid Saeed for their input and guidance.

\section{Disclosure}

The authors declare no conflicts of interest in this work.

\section{References}

1. WHO. Mental Health and Development: Targeting People with Mental Health Conditions as a Vulnerable Group. World Health Organization Publication. 2010, 1-65. Available from http://whqlibdoc.who.int/ publications/2010/9789241563949_eng.pdf?ua=1. Accessed March 13, 2014.

2. Highlights of changes from DSM-IV-TR to DSM-5. [webpage on the Internet]. http:/ www.dsm5.org/Documents/changes $\% 20$ from $\% 20 \mathrm{dsm}$ iv-tr\%20to\%20dsm-5.pdf. Accessed Jan 2014.

3. Chen CH, Lee CS, Lee MTM, et al. Variant GADL1 and response to lithium therapy in bipolar disorder. $N$ Eng $J$ Med. 2014;370: 119-128. 
4. Farooq S, Aqid O, Foussias G, Remington G. Using treatment response to subtype schizophrenia: proposal for a new paradigm in classification, Schizophr Bull. 2013;39(6):1169-1172.

5. Andreasen NC. DSM and the death of phenomenology in America: an example of unintended consequences. Schizophr Bull. 2007;33: $108-112$.

6. Stein DJ. Classification systems in psychiatry: diagnosis and global mental health in the era of DSM-5 and ICD-11. Curr Opin Psychiatry. 2013:493-497.
7. Merikangas KR, Risch N. Will the genomics revolution revolutionize psychiatry? Am J Psychiatry. 2003;160:625-635.

8. Hosak L. New findings in the genetics of schizophrenia. World J Psychiatry. 2013;3(3):57-61.

9. International Human Genome Sequencing Consortium. Finishing the euchromatic sequence of the human genome. Nature. 2004;431(7011):931-945.

10. Insel TR, Quirion R. Psychiatry as a clinical neuroscience discipline, JAMA. 2005;294(17):2221-2224.

\section{Publish your work in this journal}

Neuropsychiatric Disease and Treatment is an international, peerreviewed journal of clinical therapeutics and pharmacology focusing on concise rapid reporting of clinical or pre-clinical studies on a range of neuropsychiatric and neurological disorders. This journal is indexed on PubMed Central, the 'PsycINFO' database and CAS.
The manuscript management system is completely online and includes a very quick and fair peer-review system, which is all easy to use. Visit http://www.dovepress.com/testimonials.php to read real quotes from published authors.

Submit your manuscript here: http://www.dovepress.com/neuropsychiatric-disease-and-treatment-journal 